ఠ

\title{
Role of denileukin diftitox in the treatment of persistent or recurrent cutaneous T-cell lymphoma
}

REVIEW

\author{
Frederick Lansigan' \\ Diane M Stearns' \\ Francine Foss ${ }^{2}$ \\ 'Hematology/Oncology, Norris \\ Cotton Cancer Center, Dartmouth \\ Hitchcock Medical Center, Lebanon, \\ $\mathrm{NH}$, USA ; ${ }^{2}$ Yale Comprehensive \\ Cancer Center, Yale University School \\ of Medicine, New Haven, CT, USA
}

Correspondence: Frederick Lansigan Hematology/Oncology, Norris Cotton Cancer Center, Dartmouth Hitchcock Medical Center, One Medical Center Drive, Lebanon, NH 03755 USA $\mathrm{Tel}+\mathrm{I}$ (603) 650-5529 Email frederick.lansigan@hitchcock.org
This article was published in the following Dove Press journal:

Cancer Management and Research

5 February 2010

Number of times this article has been viewed
Abstract: Denileukin diftitox $\left(\right.$ Ontak $\left.^{\circledR}\right)$ is indicated for the treatment of patients with persistent or recurrent cutaneous T-cell lymphoma (CTCL), a rare lymphoproliferative disorder of the skin. Denileukin diftitox was the first fusion protein toxin approved for the treatment of a human disease. This fusion protein toxin combines the IL2 protein with diphtheria toxin, and targets the CD25 subunit of the IL2 receptor, resulting in the unique delivery of a cytocidal agent to CD-25 bearing T-cells. Historically, immunotherapy targeting malignant T-cells including monoclonal antibodies has been largely ineffective as cytocidal agents compared to immunotherapy directed against B-cells such as rituximab. This review will summarize the development of denileukin diftitox, its proposed mechanism of action, the pivotal clinical trials that led to its FDA approval, the improvements in quality of life, and the common toxicities experienced during the treatment of patients with CTCL. CTCL is often a chronic progressive lymphoma requiring the sequential use of treatments such as retinoids, traditional chemotherapy, or biological response modifiers. The incorporation of the immunotoxin denileukin diftitox into the sequential or combinatorial treatment of CTCL will also be addressed.

Keywords: denileukin diftitox, cutaneous T-cell lymphoma, fusion protein toxin

\section{Introduction}

Cutaneous T-cell lymphomas (CTCL) account for roughly 3\% of non-Hodgkin's lymphoma (NHL) and are a heterogenous group of disorders characterized by clonal accumulation of malignant $\mathrm{CD}^{+}$or $\mathrm{CD} 8^{+} \mathrm{T}$-lymphocytes in the skin. According to a recent review of the SEER database and other registries, the incidence of CTCL is on the rise; approximately 6.4 per million persons will be diagnosed with CTCL each year. ${ }^{1}$

Mycosis fungoides (MF) and its leukemic variant, Sézary syndrome (SS), make up the majority of CTCL. Treatment algorithms have been developed for MF and SS and include combined or sequential therapies with multiple skin-directed as well as systemic agents. ${ }^{2}$ The NCCN and EORTC also have developed guidelines in the management of MF and SS. Decisions in initial or subsequent therapies such as skindirected therapy, radiation therapy, photopheresis, anti-neoplastic therapies, biologic therapies, or combined-modality treatment incorporate the disease stage, overall prognosis, and quality-of life of the patient. For early-stage patients in whom remission is likely, the goal of therapy is to avoid long-term treatment-related toxicities; for patients with aggressive disease, approaches often include skin-directed as well as systemic therapies, including biologic or targeted therapy, and chemotherapy.

Staging of patients with MF and SS is essential both for its prognostic value and for decisions in management. The most commonly used staging system for MF/SS is based

submit your manuscript | www.dovepress.con 
on a tumor-node-metastasis-blood (TNMB) classification (Table 1). ${ }^{3}$ When MF is grouped by stage, patients with patch-plaque disease (stages IA, IB, and IIA) have an excellent survival of more than 12 years; those with tumors or erythroderma (stages IIB/III) have a median survival of approximately 4 years; however, those with stage IV which includes patients with lymph node or visceral involvement, the median survival is less than 3 years. ${ }^{4}$ Similarly, SS has a median survival of less than 3 years. ${ }^{5,6} \mathrm{Kim}$ et al showed that advanced age, $\mathrm{T}$ stage, and presence of extracutaneous disease correlated with a reduction in survival. ${ }^{7}$

Many patients die not from their disease, but the infectious complications they develop, likely due to impaired immunity as well as from immunosuppressive effects of systemic therapies. One unique feature of this disease is that as the malignant T-cell clone expands, it does so at the expense of normal T-cells, creating an immunodeficiency in numbers of functional T-cells, as well as decreased production of TH1-type cytokines, and decreased NK cell activity. ${ }^{8}$ An important treatment paradigm is to introduce treatments that do not cause further immune suppression.

Denileukin diftitox (Ontak ${ }^{\circledR}$; Eisai Inc.) is a biological therapeutic agent that is essentially a fusion protein toxin. A fusion protein toxin combines a targeting ligand such as a growth factor with a cytocidal moiety such as a plant or bacterial toxin. Denileukin diftitox uses a truncated diphtheria toxin (DAB389) as the toxophore and this is fused to the full-length sequence of the IL2 protein. The IL2 moiety of the molecule directs the fusion toxin to cells bearing IL2R. Once bound to the IL2R, denileukin diftitox is internalized via receptor-mediated endocytosis in an acidified vesicle. Upon internalization and acidification, denileukin diftitox is proteolytically cleaved within the endosomes to liberate the enzymatically active portion of the diphtheria toxin. The fragment of diphtheria toxin is translocated across the endosomal membrane into the cytosol where it inhibits protein synthesis via ADP-ribosylation of elongation factor-2, ultimately resulting in cell death., ${ }^{9,10}$

The story of the birth of denileukin diftitox is an interesting one, and it is worthwhile to review its development. Denileukin diftitox was born out of the ongoing discovery of IL2 and its receptors, and novel bioengineering.

\section{History of IL2, IL2R, and anti-Tac}

Since the cloning of the "autocrine T-cell growth factor", IL2, in 1983, the IL2 receptor (IL2R) has been the subject of major investigation. Targeting of IL2R has subsequently been explored for its therapeutic potential as well as to help elucidate signal transduction pathways. We know now that the IL2R is a complex comprised by three parts: the alpha chain (CD25) which is required for high affinity binding, the beta chain (CD122) which participates in IL2 and IL15 signal transduction, and the common gamma chain (CD132) which participates in downstream signal transduction of IL2 and other cytokines. ${ }^{11-14}$

The generation of an anti-CD25 (anti-Tac) antibody has helped identify populations of cells where IL2R is expressed..$^{15}$ In a normal subject, the IL2R is expressed mostly at a low level in less than $5 \%$ of normal circulating peripheral blood mononuclear cells (PBMC). However, the

Table I Staging system for mycosis fungoides/Sézary syndrome based on a tumor-node-metastasis-blood classification

\begin{tabular}{|c|c|c|c|c|c|}
\hline $\mathbf{N}$ & M & $\begin{array}{c}\text { TI: limited } \\
\text { patch/plaque } \\
\text { ( }<10 \% \text { BSA })\end{array}$ & $\begin{array}{l}\text { T2: generalized } \\
\text { patch/plaque } \\
(\geq 10 \% \text { BSA })\end{array}$ & T3 tumor & $\begin{array}{c}\mathrm{T4} \\
\text { erythroderma }\end{array}$ \\
\hline $\begin{array}{l}\text { No: nodes } \\
\text { clinically uninvolved }\end{array}$ & Mo & $\mathrm{IA}$ & IB & \multirow{2}{*}{ IIB } & IIIA \\
\hline $\begin{array}{l}\text { NI: nodes enlarged, } \\
\text { histologically uninvolved }\end{array}$ & Mo & \multicolumn{2}{|c|}{ IIA } & & IIIB \\
\hline $\begin{array}{l}\text { N2-3: nodes } \\
\text { clinically normal (N2) } \\
\text { or enlarged (N3), } \\
\text { histologically involved }\end{array}$ & Mo & \multicolumn{4}{|c|}{ IVA } \\
\hline $\begin{array}{l}\text { N0-3: visceral } \\
\text { involvement }\end{array}$ & MI & \multicolumn{4}{|c|}{ IVB } \\
\hline \multicolumn{6}{|c|}{ B0 Absence of significant peripheral blood Sézary cells } \\
\hline \multicolumn{6}{|c|}{ BI Low tumor burden that does not meet the criteria of B2 cells } \\
\hline \multicolumn{6}{|c|}{ B2 Significant peripheral blood $>1000 / \mu$ L Sézary cells with positive clone } \\
\hline
\end{tabular}

Abbreviation: BSA, body surface area. 
greatest level of expression of CD25 is seen in the setting of T-cell malignancies such as cutaneous T-cell lymphoma, and adult T-cell leukemia. It is also highly expressed in hairy cell leukemia, Hodgkin's disease, as well as some B-cell NHLs and histiocytic proliferations. Such observations are the basis for therapies that target IL2R-expressing cells. Indeed, high- or intermediate affinity IL2R isoforms are expressed in at least $50 \%$ of CTCL and $25 \%$ to $50 \%$ of B-cell non-Hodgkin's lymphomas when tissue is examined by flow cytometry, and thus make the IL2R an attractive target for therapy. ${ }^{16}$

In 1995 Sakaguchi et al discovered that the high affinity IL2 receptor alpha chain was a reliable cell surface marker for regulatory T-cells (Tregs). ${ }^{17} \mathrm{CD} 4{ }^{+} \mathrm{CD} 25^{+}$cells were found to be anergic, failing to proliferate when stimulated by antiCD3 antibodies. They also suppressed the growth of CD4 ${ }^{+}$ CD25- T-cells in vitro, as well as an admixture of conventional T-cells. Interestingly, although IL2 was required for their growth and survival, Tregs did not produce IL2 themselves. ${ }^{18}$ Since CTCL is considered by some to be a malignant proliferation of T-regs, ${ }^{19}$ the rationale behind targeting CD25 is even more robust.

Abnormalities of IL2R were also detected in autoimmune diseases such as rheumatoid arthritis, systemic lupus erythematosus, aplastic anemia, and insulin-dependent diabetes. Increased expression of CD25 was also detected in the serum of patients during solid organ allograft rejection, and in those with graft-versus-host disease (GVHD). Since the cloning of the IL2R receptor, work has been done to exploit the receptor as a target for therapy in T-cell malignancies, GVHD, and other autoimmune diseases.

Daclizumab is a humanized anti-Tac monoclonal antibody that has been tested in leukemia and lymphoma. In cell lines, it was thought that daclizumab blocked the interaction of IL2 and its receptor resulting in cytokine-deprivationmediated apoptotic cell death. ${ }^{20}$ Additionally, based on in vivo data from mouse models, it was thought that daclizumab also exerted an effect through an FcR receptor mechanism. ${ }^{21}$

Clinical trials using daclizumab in adult T-cell leukemia and lymphoma (ATL) was associated with partial responses predominantly in patients with smoldering or chronic ATL. Responding patients were hypothesized to have a blockage of the IL2-IL2R autocrine pathway.

Unfortunately, unlike other therapeutic monoclonal antibodies used in the treatment of human malignancies, daclizumab did not seem to have strong cytocidal activity. Therefore, other strategies were needed to make use of IL2R targeting in T-cell malignancies.

\section{The IL2R-diphtheria toxin fusion protein: DAB486-IL2}

In order to target the delivery of a cytocidal moiety to IL2Rbearing cells, human IL2 was fused to truncated diphtheria toxin (DT). Unlike daclizumab (anti-Tac), which is a mAb directed against the CD25 component of the receptor, DAB486-IL2 contained the full-length sequence of IL2 as the ligand for the IL2R. The original fusion protein toxin used a fragment of DT containing methionine plus the first 485 amino acids of DT resulting in the molecule DAB486IL2., ${ }^{9,22,23}$ While this molecule had clinical activity (1 complete response, 2 partial responses, out of 18 patients), the maximum tolerated dose (MTD) was only $0.1 \mathrm{mg} / \mathrm{kg} /$ day, due to transaminase elevations. ${ }^{24}$ Five of 18 patients experienced fever associated with administration of the fusion protein toxin that responded to acetaminophen. The anti-tumor effect of DAB486-IL2 did not correlate with pre- or post-treatment antibodies to DT, DAB486-IL2, or IL2.

\section{Denileukin diftitox (DAB 389-IL2)}

Modifications were made to DAB 486-IL2 by removing 97 amino acids from the native part of the toxin containing the disulfide bond, resulting in an increase in half-life and tolerance in animals. This resulted in a 5-fold greater affinity for IL2R and a 10-fold increase in potency. ${ }^{25,26}$ The resulting DAB389-IL2 fusion protein toxin is otherwise known as denileukin diftitox.

Denileukin diftitox binds to IL2R in the same way as IL2 because it contains the full-length sequence of IL2. This optimized fusion protein toxin binds to a high- or intermediateaffinity receptor. ${ }^{27,28}$ Intermediate affinity receptors have beta (CD122) and gamma (CD132) subunits and bind IL2 with $\mathrm{Kd} \sim 10^{-9}$, and high-affinity receptors have the addition of the alpha (CD25) subunit and bind IL2 with $\mathrm{Kd} \sim 10^{-11}$. Once denileukin diftitox binds to IL2R, this molecule is internalized into the cell where the truncated diphtheria toxin is cleaved off, liberated into the cytosol, and induces cell death by causing apoptosis.

A phase I clinical trial using DAB389-IL2 in patients with lymphomas expressing IL2R (CD25 or CD122) resulted in an MTD of $27 \mu \mathrm{g} / \mathrm{kg} /$ day. ${ }^{25}$ Unlike with the first generation fusion protein toxin, changes in liver function tests were not dose-limiting. The dose-limiting toxicities that were seen included asthenia, fever, nausea and vomiting. In addition, some of the CTCL patients who were treated in this trial exhibited signs and symptoms of capillary leak syndrome with edema, hypotension and hypoalbuminemia. Again, the 
presence of antibodies to DT, DAB389-IL2, or IL2 pre- and post-treatment did not prevent treatment responses. The half-life of DAB 389-IL2 was found to be 72 minutes with monophasic clearance, and there was no dose accumulation with repeated doses of the drug.

In this trial, 35 patients had CTCL (30 MF, 4 large cell CTCL, and 1 cutaneous PTCL), and most were heavily pretreated. Of the 35 CTCL patients, 4 (11\%) had a complete response, and $8(24 \%)$ had a partial response. The median time to response was 2 months and the duration response ranged from 2 to $39+$ weeks (median 10 months). There was no obvious correlation with the expression of CD122 or CD132. ${ }^{25}$

In another phase I/II trial using DAB 389-IL2 that enrolled a more homogenous group of 35 patients with MF, a dose and schedule of DAB 389-IL2 given over 5 consecutive days of a 21-day cycle was explored. Thirteen patients (37\%) achieved an objective response, including a complete response in 5 patients (14\%). Complete response was achieved in patients with extensive erythroderma and tumor-stage mycosis fungoides. ${ }^{29}$

A smaller trial of 4 heavily pre-treated CTCL patients who received either 9 ( 1 patient) or 18 (3 patients) $\mu \mathrm{g} / \mathrm{kg} / \mathrm{day}$ of DAB 389-IL2 for 5 days of a 21-day cycle showed striking results. ${ }^{30}$ One patient achieved a complete pathological response, and another patient had a complete clinical response, and the other 2 patients had partial responses. Similar to prior trials, the duration of response ranged from 5 to $11+$ months. There was significant improvement in the symptoms and quality of life in these patients.

These trials formed the basis of the pivotal phase III trial of denileukin diftitox. ${ }^{31}$ This randomized, blinded, multicenter, parallel group study was designed to evaluate the efficacy, safety, and pharmacokinetics of two doses of denileukin diftitox in patients with previously treated, advanced or refractory CTCL. The inclusion criteria required that patients have $\geq 20 \%$ of lymphocytes within the skin biopsy stain positive for CD25. Patients were randomized to receive either denileukin diftitox 9 or $18 \mu \mathrm{g} / \mathrm{kg} /$ day administered on 5 consecutive days every 3 weeks for up to 8 cycles, and were stratified by stage (ie, $\leq$ IIA or $\geq$ IIB). All patients were monitored for toxicity and clinical efficacy, the latter being assessed by changes in disease burden and quality of life measurements.

The study enrolled 71 patients, and 48 (68\%) had advanced disease (stage IIB or greater). Overall, 21 (30\%) of the 71 patients with CTCL treated with denileukin diftitox had an objective response (20\% partial response and $10 \%$ complete response). There was no difference in the overall response rate between the 9 or $18 \mu \mathrm{g} / \mathrm{kg} /$ day cohorts; however, a subset analysis of patients with stage IIB disease or higher, showed a trend towards higher response $(P=0.07)$. Quality-of-life improvements following denileukin diftitox therapy were seen both in responders and in nonresponders, and this was mainly a result of clinical improvements in pruritus in $68 \%$ of the patients. In patients who responded, the use of rescue medications such as topical steroids or oral antihistamines was also decreased.

Fifty of the 71 patients were classified as nonresponders; however only $2(3 \%)$ of patients had progression of disease. The remainder of the patients had stable disease $(32 \%)$, or withdrew due to intolerance to the therapy (35\%) before they could be assessed. The authors commented that even in patients who were classified as stable disease, had a significant initial response to therapy.

It is again noteworthy that the majority of patients had detectable levels of antidenileukin diftitox and/or antiIL2 after treatment with denileukin diftitox, however, the presence of these antibodies did not impair the response to treatment. Curiously, higher antidenileukin diftitox antibody levels were associated with a lower incidence of rash, hypoalbuminemia, and transaminase elevations, but did not correspond to infusion-related reactions. It is thought that the development of antibodies to denileukin diftitox may contribute to the increase in clearance of denileukin diftitox that was observed by cycle 3 .

Negro-Vilar et al reported preliminary results of a placebo-controlled phase III trial of up to 8 cycles of denileukin diftitox in 144 patients with stage IA to III disease, the largest CTCL trial to date. ${ }^{32}$ Patients with at least 3 prior treatments were included. Overall response rates for the placebo, low-dose $(9 \mu \mathrm{g} / \mathrm{kg})$, and high-dose $(18 \mu \mathrm{g} / \mathrm{kg})$ treatment arms were $16 \%, 38 \%$, and $49 \%$, respectively, with median times to progression of 4,26 , and greater than 32 months, respectively. In an unpublished meta-analysis of three phase III clinical trials of denileukin diftitox for the treatment of CTCL, Negro-Vilar also reported there was no correlation between CD25 expression and response. ${ }^{33}$

\section{Denileukin diftitox in combination treatments}

Since the expression of IL2R has been largely thought to be important in T-cell targeting of denileukin diftitox, strategies to upregulate IL2R expression have been explored. Retinoids have demonstrated efficacy in the treatment of MF/SS and have antiproliferative, antiangiogenic, immune-modulating, 
and cellular effects. ${ }^{34}$ In vitro studies demonstrated that the retinoid $\times$ receptor $(\mathrm{RXR})$ retinoid, bexarotene, at biologically relevant concentrations of $10(-6) \mathrm{M}$ to $10(-8) \mathrm{M}$, upregulated both the p55 and p 75 subunits of the IL2R and the susceptibility of T-cell leukemia cells to denileukin diftitox..$^{35,36}$ In a phase I dose-escalation trial, denileukin diftitox $18 \mu \mathrm{g} / \mathrm{kg} \times 3$ days was administered in combination with oral bexarotene ( 75 to $300 \mathrm{mg} /$ day) every 21 days to patients with relapsed or refractory $\mathrm{MF} / \mathrm{SS} .{ }^{36}$ The overall response rate was $70 \%$, and responders included patients who had stable disease or no response on denileukin diftitox alone. CD25 expression on $\mathrm{CD}^{+}$cells was measured by immunohistochemistry or flow cytometry at baseline and after 7 days of bexarotene. Modulation of the IL2R was seen with doses of bexarotene $150 \mathrm{mg}$ daily and higher. All 4 patients who achieved a CR and 1 of 3 patients who achieved a PR had evidence of $>50 \%$ upregulation after bexarotene treatment. The combination was tolerated well with no overlapping adverse events.

Arginine butyrate is a histone deacetylase (HDAC) inhibitor that also has been shown to increase IL2R-beta (CD122) subunit gene and protein expression. ${ }^{37}$ While low dose arginine butyrate showed no growth inhibitor effect of its own, combination studies with denileukin diftitox in vitro showed an increase in cytotoxicity. Since HDAC inhibitors are clinically active compounds in CTCL, combination therapies to test potential synergism with denileukin diftitox are warranted.

\section{Improvements of quality of life}

CTCL affects numerous dimensions of quality of life (QOL) due to relentless painful, pruritic and disfiguring lesions. QOL was evaluated as a secondary endpoint in heavily pretreated advanced and/or recurrent CTCL patients enrolled in the multicenter randomized phase III trial of denileukin diftitox by Olsen et al using the Functional Assessment of Cancer Therapy-General (FACT-G) scale. ${ }^{31,38}$ FACT-G was developed to measure health-related QOL in patients with cancer. ${ }^{39}$ This tool measures four domains of quality of life: physical, functional, social and emotional well-being, as well as patient-physician relationships. The physical well-being scale assesses energy level, pain and side effects of treatment. Relationships with family and friends are addressed in the social well-being subscale. The emotional well-being subscale evaluates for anxiety and depression. The ability to sleep, work and participate in desired leisure activities are addressed in the functional well-being subscale. The relationship-with-physician subscale measures satisfaction with interaction and care provided by the physician.
In the study by Olsen et al the 71 patients who received denileukin diftitox were administered the FACT-G questionnaire at baseline prior to the start of therapy and prior to each treatment cycle. In addition, along with each FACT-G measurement, patients were asked to rate the appearance of their skin on a 7-point global severity scale. Because the symptom of pruritus was not specifically addressed in the physical well-being subscale of the FACT-G, patients were also asked to rate severity of pruritus on a $10-\mathrm{cm}$ visual analog scale..$^{38}$

In the 21 patients who achieved a complete or partial response to denileukin diftitox, the FACT-G and most individual subscale scores improved during the study period reaching statistical significance by cycle $3(P<0.05)$ and were significantly higher than scores of non-responders at the completion of the study $(P<0.041) .{ }^{38}$ Additionally for responders, skin and pruritus severity scores showed significant improvement at study end compared with baseline measurements. Nonresponders also showed brief improvement in QOL while receiving denileukin diftitox, but this declined with the discontinuation of therapy.

\section{Management of toxicities}

Overall, the most commonly reported adverse events (AE) in the pivotal study by Olsen et al were flu-like symptoms $(85 \%)$. These reactions generally improved with an antipyretic, antiemetic, and/or antidiarrheal treatment. Other common AEs were acute hypersensitivity-type reactions, asthenia, nausea/vomiting (which led to dehydration in some patients), and infectious complications. The frequency of hypersensitivity reactions that occurred during or within 24 hours of drug administration was $60 \%$ and improved with steroid, antihistamine, and acetaminophen pre-medication prior to subsequent cycles. It should be noted that routine pre-medication was prohibited in this study. In clinical use, the routine use of pre-medication does reduce this complication significantly, however. The incidence of serious infusion reactions across 3 clinical studies ( $\mathrm{n}=234$ patients) was $8.1 \%$. Various pre-medication protocols have been proposed including one by Dang et $\mathrm{al}^{40}$ and consists of dexamethasone $4 \mathrm{mg}$ iv, diphenhydramine 25 to $50 \mathrm{mg}$ iv, and acetaminophen $650 \mathrm{mg}$ po.

Vascular leak syndrome was reported in $25 \%$ of patients and was defined as hypotension, hypoalbuminemia, and peripheral edema. The vascular leak syndrome occurred within the first 14 days of treatment and was usually selflimited. Patients responded well to judicious use of diuretics for edema, and slow hydration for hypotension. Serum 
albumin $<3 \mathrm{~g} / \mathrm{dL}$ and pre-exisiting edema seemed to predict the development of vascular leak syndrome. The authors recommend an additional clinical evaluation of the patient for changes in weight and fluid shifts at least one time within the first 10 days after treatment begins. The US Food and Drug Administration boxed warning states that denileukin diftitox should only be administered in a facility equipped and staffed for cardiopulmonary resuscitation.

All adverse events in the pivotal phase III study were most severe during the first two cycles of treatment and then diminished significantly. Hepatic transaminase levels were commonly elevated, with $19 \%$ of patients experiencing grade 3-4 elevations. Most elevations were observed during the first therapy cycle and diminished during all subsequent cycles. Mild elevations in creatinine were noted rarely and were reversible. There was no significant bone marrow suppression, although lymphocyte counts fluctuated transiently in $70 \%$ of patients. Infections were reported but were considered typical infections of CTCL patients, and not related to denileukin diftitox.

Postmarketing reports of serious events include thyrotoxicosis as reported by Ghori et $\mathrm{al}^{41}$ Clinical thyrotoxicosis was described in 8 patients treated at a single center; 4 patients developed thyrotoxicosis within days of the first cycle of denileukin diftitox therapy, and 4 patients after the second cycle. Symptoms of tremor, tachycardia, nausea, diarrhea, and weight loss should trigger suspicion of this condition which resolves after drug discontinuation. Subsequent clinical hypothyroidism developed in 5 of these patients requiring thyroid replacement therapy. Loss of visual acuity and color vision is another black box warning and may have to do with retinal changes, however the mechanism of this rare phenomenon is not known. ${ }^{42}$ Lastly, rhabdomyolysis has been reported in the context of severe vascular leak, ${ }^{43}$ and the authors also have independently experienced this complication in two of their patients.

\section{Conclusion}

Denileukin diftitox has made great strides in the treatment of CTCL since its development 20 years ago. The reported response rates range from $30 \%$ to $50 \%$, and treatment definitely improves the quality of life of these patients. However, some questions still remain.

The optimal time to use denileukin diftitox is not well defined. The toxicity profile and unfavorable schedule of administration have prevented its use as upfront treatment, however, denileukin diftitox may be more effective if used earlier in the course of the disease. While the initial response rate of denileukin diftitox was 38\% in early-stage CTCL, the median number of prior treatments was five. In less heavily treated patients, however, the response rate was considerably higher, $63 \%$ and $48 \%$, for early and late stage CTCL, respectively. This suggests that the response rate may be higher in patients who are treated earlier in the course of their disease.

We are only now beginning to understand how to utilize denilekin diftitox in combination therapy. A combination trial of denileukin diftitox with bexarotene has shown increased activity over monotherapy. Due to non-overlapping toxicity profiles, denileukin diftitox has also been successfully been combined with CHOP (cyclophosphamide, doxorubin, vincristine and prednisone) for peripheral T-cell lymphoma, suggesting that chemotherapy combinations that incorporate this immunotoxin may eventually have a place in the treatment of advanced CTCL. It would also be useful to predict which patients would benefit most from treatment with denileukin diftitox. Initally, it was thought that the presence of CD25 was required for entry of the drug into the malignant T-cells, however, recent evidence has shown durable responses in patients with CD25- disease. Regardless of these limitations, this unique immunotoxin has proven itself effective for the treatment of CTCL, a chronic disease with considerable morbidity and mortality.

\section{Disclosures}

The authors disclose no conflicts of interest.

\section{References}

1. Criscione VD, Weinstock MA. Incidence of cutaneous T-cell lymphoma in the United States, 1973-2002. Arch Dermatol. 2007;143:854-859.

2. Lansigan F, Choi J, Foss FM. Cutaneous T-cell lymphoma. Hematol Oncol Clin North Am. 2008;22:979-996, x.

3. Bunn PAJ, Lamberg, SI. Report of the Committee on Staging and Classification of Cutaneous T-Cell Lymphomas. Cancer Treat Rep. 1979;63:725-728

4. Sausville EA, Eddy JL, Makuch RW, et al. Histopathologic staging at initial diagnosis of mycosis fungoides and the Sezary syndrome. Definition of three distinctive prognostic groups. Ann Intern Med. 1988;109:372-382.

5. Demierre MF, Kim YH, Zackheim HS. Prognosis, clinical outcomes and quality of life issues in cutaneous T-cell lymphoma. Hematol Oncol Clin North Am. 2003;17:1485-1507.

6. Vonderheid EC, Bernengo MG, Burg G, et al. Update on erythrodermic cutaneous T-cell lymphoma: report of the International Society for Cutaneous Lymphomas. J Am Acad Dermatol. 2002;46:95-106.

7. Kim YH, Liu HL, Mraz-Gernhard S, Varghese A, Hoppe RT. Longterm outcome of 525 patients with mycosis fungoides and Sezary syndrome: clinical prognostic factors and risk for disease progression. Arch Dermatol. 2003;139:857-866.

8. Heald P. Probing the immune dysregulation in cutaneous T-cell lymphomas. J Invest Dermatol. 2005;125:xvi-xvii.

9. Bacha P, Williams DP, Waters C, Williams JM, Murphy JR, Strom TB. Interleukin 2 receptor-targeted cytotoxicity. Interleukin 2 receptormediated action of a diphtheria toxin-related interleukin 2 fusion protein. J Exp Med. 1988;167:612-622. 
10. Foss FM. DAB(389)IL-2 (denileukin diftitox, ONTAK): a new fusion protein technology. Clin Lymphoma. 2000;1 Suppl 1:S27-31.

11. Hatakeyama M, Minamoto S, Uchiyama T, Hardy RR, Yamada G, Taniguchi T. Reconstitution of functional receptor for human interleukin-2 in mouse cells. Nature. 1985;318:467-470.

12. Leonard WJ, Depper JM, Crabtree GR, et al. Molecular cloning and expression of cDNAs for the human interleukin-2 receptor. Nature. 1984;311:626-631.

13. Takeshita T, Asao H, Ohtani K, et al. Cloning of the gamma chain of the human IL-2 receptor. Science. 1992;257:379-382.

14. Tsudo M, Kitamura F, Miyasaka M. Characterization of the interleukin 2 receptor beta chain using three distinct monoclonal antibodies. Proc Natl Acad Sci U S A. 1989;86:1982-1986.

15. Morris JC, Waldmann TA. Advances in interleukin 2 receptor targeted treatment. Ann Rheum Dis. 2000;59 Suppl 1:i109-i114.

16. Foss F. Clinical experience with denileukin diftitox (ONTAK). Semin Oncol. 2006;33:S11-S16.

17. Sakaguchi S, Sakaguchi N, Asano M, Itoh M, Toda M. Immunologic selftolerance maintained by activated T-cells expressing IL-2 receptor alphachains (CD25). Breakdown of a single mechanism of self-tolerance causes various autoimmune diseases. J Immunol. 1995;155:1151-1164.

18. Randolph DA, Fathman CG. Cd4+ Cd25+ regulatory T-cells and their therapeutic potential. Annu Rev Med. 2006;57:381-402.

19. Berger CL, Tigelaar R, Cohen J, et al. Cutaneous T-cell lymphoma: malignant proliferation of T-regulatory cells. Blood. 2005;105:1640-1647.

20. Depper JM, Leonard WJ, Kronke M, Waldmann TA, Greene WC. Augmented T-cell growth factor receptor expression in HTLV-1-infected human leukemic T-cells. J Immunol. 1984;133:1691-1695.

21. Zhang M, Zhang Z, Garmestani K, et al. Activating Fc receptors are required for antitumor efficacy of the antibodies directed toward CD25 in a murine model of adult t-cell leukemia. Cancer Res. 2004;64:5825-5829.

22. Bacha P, Forte S, Kassam N, et al. Pharmacokinetics of the recombinant fusion protein DAB486IL-2 in animal models. Cancer Chemother Pharmacol. 1990;26:409-414.

23. Williams DP, Parker K, Bacha P, et al. Diphtheria toxin receptor binding domain substitution with interleukin-2: genetic construction and properties of a diphtheria toxin-related interleukin-2 fusion protein Protein Eng. 1987;1:493-498.

24. LeMaistre CF, Meneghetti C, Rosenblum M, et al. Phase I trial of an interleukin-2 (IL-2) fusion toxin (DAB486IL-2) in hematologic malignancies expressing the IL-2 receptor. Blood. 1992;79:2547-2554.

25. LeMaistre CF, Saleh MN, Kuzel TM, et al. Phase I trial of a ligand fusion-protein (DAB389IL-2) in lymphomas expressing the receptor for interleukin-2. Blood. 1998;91:399-405.

26. Williams DP, Snider CE, Strom TB, Murphy JR. Structure/function analysis of interleukin-2-toxin (DAB486-IL-2). Fragment B sequences required for the delivery of fragment A to the cytosol of targeT-cells. J Biol Chem. 1990;265:11885-11889.

27. Attia P, Maker AV, Haworth LR, Rogers-Freezer L, Rosenberg SA Inability of a fusion protein of IL-2 and diphtheria toxin (Denileukin Diftitox, DAB389IL-2, ONTAK) to eliminate regulatory T-lymphocytes in patients with melanoma. $J$ Immunother. 2005;28:582-592.
28. Talpur R, Jones DM, Alencar AJ, et al. CD25 expression is correlated with histological grade and response to denileukin diftitox in cutaneous T-cell lymphoma. J Invest Dermatol. 2006;126:575-583.

29. Saleh MN, LeMaistre CF, Kuzel TM, et al. Antitumor activity of DAB389IL-2 fusion toxin in mycosis fungoides. J Am Acad Dermatol. 1998;39:63-73.

30. Duvic M, Cather J, Maize J, Frankel AE. DAB389IL2 diphtheria fusion toxin produces clinical responses in tumor stage cutaneous T-cell lymphoma. Am J Hematol. 1998;58:87-90.

31. Olsen E, Duvic M, Frankel A, et al. Pivotal phase III trial of two dose levels of denileukin diftitox for the treatment of cutaneous T-cell lymphoma. J Clin Oncol. 2001;19:376-388.

32. Negro-Vilar A, Dziewanowska Z, Groves ES, et al. Efficacy and safety of denileukin diftitox (Dd) in a phase III, double-blind, placebo-controlled study of CD25+ patients with cutaneous T-cell lymphoma (CTCL). J Clin Oncol (Meeting Abstracts). 2007;25:8026-.

33. Negro-Vilar A, Prince HM, Duvic M, Richardson S, Sun Y, Acosta M. Efficacy and safety of denileukin diftitox (Dd) in cutaneous T-cell lymphoma (CTCL) patients: Integrated analysis of three large phase III trials. J Clin Oncol 26:2008 May 20 suppl; abstr 8551.

34. Mahrle G, Thiele B. Retinoids in cutaneous T-cell lymphomas. Dermatologica. 1987;175 Suppl 1:145-150.

35. Gorgun G, Foss F. Immunomodulatory effects of RXR rexinoids: modulation of high-affinity IL-2R expression enhances susceptibility to denileukin diftitox. Blood. 2002;100:1399-1403.

36. Foss F, Demierre MF, DiVenuti G. A phase-1 trial of bexarotene and denileukin diftitox in patients with relapsed or refractory cutaneous T-cell lymphoma. Blood. 2005;106:454-457.

37. Shao RH, Tian X, Gorgun G, Urbano AG, Foss FM. Arginine butyrate increases the cytotoxicity of DAB(389)IL-2 in leukemia and lymphoma cells by upregulation of IL-2Rbeta gene. Leuk Res. 2002;26:1077-1083.

38. Duvic M, Kuzel TM, Olsen EA, et al. Quality-of-life improvements in cutaneous T-cell lymphoma patients treated with denileukin diftitox (ONTAK). Clin Lymphoma. 2002;2:222-228.

39. Cella DF, Tulsky DS, Gray G, et al. The Functional Assessment of Cancer Therapy scale: development and validation of the general measure. J Clin Oncol. 1993;11:570-579.

40. Dang NH, Fayad L, McLaughlin P, et al. Phase II trial of the combination of denileukin diftitox and rituximab for relapsed/refractory B-cell non-Hodgkin lymphoma. Br J Haematol. 2007;138:502-505.

41. Ghori F, Polder KD, Pinter-Brown LC, et al. Thyrotoxicosis after denileukin diftitox therapy in patients with mycosis fungoides. $J$ Clin Endocrinol Metab. 2006;91:2205-2208.

42. Ruddle JB, Harper CA, Honemann D, Seymour JF, Prince HM A denileukin diftitox (Ontak) associated retinopathy? Br J Ophthalmol. 2006;90:1070-1071

43. Avarbock AB, Loren AW, Park JY, et al. Lethal vascular leak syndrome after denileukin diftitox administration to a patient with cutaneous gamma/delta T-cell lymphoma and occult cirrhosis. Am J Hematol. 2008;83:593-595.
Cancer Management and Research

\section{Publish your work in this journal}

Cancer Management and Research is an international, peer-reviewed open access journal focusing on cancer research and the optimal use of preventative and integrated treatment interventions to achieve improved outcomes, enhanced survival and quality of life for the cancer patient. The journal welcomes original research, clinical \& epidemiological

\section{Dovepress}

studies, reviews \& evaluations, guidelines, expert opinion \& commentary, case reports \& extended reports. The manuscript management system is completely online and includes a very quick and fair peerreview system, which is all easy to use. Visit http://www.dovepress.com/ testimonials.php to read real quotes from published authors. 